\title{
Recomendação de Recursos Educacionais para Grupos: buscando soluções em Redes Sociais
}

\author{
Rafael F. Almeida, Crystiam Kelle Pereira, Fernanda Campos, Victor Stroele \\ Universidade Federal de Juiz de Fora (UFJF) - Programa de Pós-Graduação em Ciência \\ da Computação - Núcleo de Pesquisa em Engenharia do Conhecimento \\ Juiz de Fora - MG - Brasil \\ rafaelfalmeida@gmail.com, crystiamkelle@gmail.com, \\ fernanda.campos@ufjf.edu.br, victor.stroeleaice.ufje.br
}

\begin{abstract}
A recommender system must generate satisfactory recommendations, so it is important to identify information that helps to define user's profile, like as a result of interactions that occur in social networks. This work aims to explore the information generated by a users group on social networks in the search and selection process of educational resources, considering their members common interest. The evaluation of the proposal consists of a case study, and the results point to the viability of the solution.
\end{abstract}

Resumo. Para que um Sistema de Recomendação consiga gerar recomendações satisfatórias, é importante identificar informações que ajudem a definir o perfil do usuário, como, por exemplo, em decorrência das interações que ocorrem nas redes sociais. Este trabalho visa explorar as informações geradas por um grupo de usuário nas redes sociais no processo de busca e seleção de recursos educacionais de interesse comum de seus membros. A avaliação da proposta foi composta de um estudo de caso e os resultados apontam para a viabilidade da solução.

\section{Introdução}

As dificuldades de localização dos recursos educacionais, bem como as restrições dos repositórios de objetos de aprendizagem, têm grande impacto na eficiência dos Sistemas de Recomendação (SR) e de personalização de conteúdo educacional. Em meio à sobrecarga de informação, os SR coletam informações sobre as preferências de seus usuários para um conjunto de itens, visando o auxílio aos usuários no processo de busca e acesso a informações relevantes (Rodrigues et al., 2015).

A implementação de SR ocorre em diversas áreas e os trabalhos mais comuns estão focados em recomendação de filmes, músicas, televisão, livros, documentos, elearning, e-commerce, aplicações em mercados e de busca na web, entre outros (Park et al., 2012). Com isso, foram adotados SR com o objetivo de complementar a capacidade dos sistemas de busca tradicionais (Carvalho e Macedo, 2014). SR tradicionalmente recomendam itens para usuários individuais. Em alguns cenários, entretanto, a recomendação para um grupo de indivíduos é mais adequada. Um dos grandes desafios desses sistemas é como lidar adequadamente com as preferências de cada integrante do grupo para geração de recomendação conjunta (Yu, 2012). 
O uso de redes sociais é uma prática bastante comum entre os brasileiros e caracteriza o perfil de estudantes usuários de Internet. De acordo com a TIC Educação 2014, o Facebook é utilizado por $97 \%$ dos alunos que participam de redes sociais. É interessante observar que, entre os alunos que participam de redes sociais, apenas $28 \%$ dizem não as usar para trabalhos escolares (CGI, 2015).

Este trabalho explora as informações geradas por grupos de usuários nas redes sociais, de forma a auxiliar o processo de busca e seleção de recursos educacionais, através de um Sistema de Recomendação para Grupos. A proposta prevê a recomendação para o grupo levando em consideração as características individuais dos seus membros, podendo assim, atender a um número maior de pessoas ao mesmo tempo. Nesse sentido o BROAD-GRS (Sistema de Recomendação para Grupos) avança em relação aos trabalhos anteriores do Projeto BROAD (Pereira et al., 2014) (Pereira et al., 2015a) (Pereira et al., 2015b) (Rezende et al., 2015).

Este artigo está organizado da seguinte forma: a seção 2 apresenta a metodologia da pesquisa e a seção 3 os trabalhos relacionados. Já a seção 4 descreve a proposta da arquitetura para a construção do BROAD-GRS. A seção 5 apresenta o protótipo desenvolvido enquanto a seção 6 apresenta a avaliação da proposta. Por fim, a seção 7 apresenta as considerações finais.

\section{Metodologia da Pesquisa}

A metodologia utilizada neste trabalho inclui (1) a utilização de uma revisão sistemática; (2) a proposta e o desenvolvimento de uma arquitetura capaz de extrair características de grupos de usuários em redes sociais e realizar recomendações de recursos educacionais, condizentes com essas características, aos membros do grupo; e (3) a avaliação através do desenvolvimento de um protótipo e de um estudo de caso. A utilização da revisão sistemática realizada em (Almeida et al., 2015) teve como objetivo identificar estudos sobre Sistemas de Recomendação para grupos em redes sociais. O resultado serviu de base para a construção da arquitetura. Para validar a proposta, foi desenvolvido um protótipo utilizando a rede social Facebook. A recomendação é feita através de três abordagens: baseada em repositórios de recursos educacionais, em repositórios de Dados Ligados e baseada em um repositório de vídeos. Visando avaliar a viabilidade e eficiência da utilização da proposta deste trabalho, foi realizado um estudo de caso (Wohlin et al., 2012).

\section{Trabalhos Relacionados}

Em (Ribeiro et al., 2013) é apresentado um sistema para recomendação de Objetos de Aprendizagem aos alunos do AVA Moodle, baseado no conjunto das hashtags atribuídas nas postagens dos fóruns, fazendo uso do esquema da frequência de ocorrência do termo no documento e do inverso da frequência do termo entre documentos da coleção. Foi desenvolvido um sistema que permite localizar o conjunto de hashtags mais postadas nos fóruns da plataforma, classificando-as, ranqueando-as e sugerindo conteúdos relacionados ao tema em estudo. Já em (Casagrande et al., 2013) foi proposta uma técnica de recomendação de conteúdos em repositórios digitais baseada em metadados descritores dos conteúdos e agrupamento de usuários. O perfil do usuário é construído implicitamente observando os valores dos metadados dos 
conteúdos acessados. A proposta foi implementada na Biblioteca Digital de Literatura Brasileira, com a qual foram realizados testes de precisão e de tempo de processamento. A atividade final teve por objetivo produzir uma lista ordenada de conteúdos a serem recomendados a um determinado usuário. Esta lista é obtida a partir do conjunto de conteúdos acessados pelos vizinhos próximos do usuário foco da recomendação. Esta técnica assume que a lista de recomendação deve ser constituída de conteúdos que não foram ainda acessados pelo usuário. (Pereira et al., 2015a) busca extrair e explorar as informações disponíveis nas redes sociais, bem como o interesse dos seus usuários em estarem atualizados através do seu uso. Por meio da extração de informações disponíveis nas redes sociais, buscam identificar e inferir características, preferências e interesses educacionais dos usuários, usando técnicas de Extração de Texto e Web Semântica. O objetivo principal é a identificação de características do perfil e do contexto do usuário a partir de informações geradas, espontaneamente, através do uso das redes sociais, para auxiliar no processo de seleção e recomendação de recursos educacionais adequados a esse perfil. O sistema de recomendação proposto se diferencia ao abordar a recomendação de recursos educacionais, no ambiente de redes sociais, e, tendo um grupo de usuários como público alvo, modelo não identificado em outros trabalhos durante a revisão sistemática (Almeida et al., 2015).

\section{Arquitetura BROAD-GRS}

O foco dessa proposta é a definição do perfil e do contexto dos grupos de usuários, através da aquisição de informações disponibilizadas nas redes sociais por meio de um Sistema de Recomendação de recursos educacionais para Grupos. A proposta explora o longo tempo dispendido por usuários nas redes sociais e recursos como "recomendar", "curtir", "compartilhar", "comentar", que podem aumentar a propagação das recomendações educacionais e a qualidade dos conteúdos educacionais distribuídos.

No escopo deste trabalho, Rede Social é uma das formas de representação dos relacionamentos afetivos ou profissionais dos seres entre si ou entre seus agrupamentos de interesses mútuos. A rede é responsável pelo compartilhamento de ideias entre pessoas que possuem interesses e objetivos em comum e também valores a serem compartilhados. Assim, um grupo é composto por indivíduos que possuem interesses em comum. Neste trabalho, utilizamos a rede social Facebook, pois, segundo (Junco, 2011), é o site de rede social mais popular entre estudantes universitários.

Para processar as informações extraídas, definir características de perfil do grupo e gerar recomendações educacionais aderentes a essas características definiu-se a arquitetura conceitual (Figura 1) dividida em 5 módulos: (1) Camada de extração de informações; (2) Camada de definição do perfil do grupo; (3) Camada de representação semântica; (4) Camada de recomendação e (5) Camada de interface.

\subsection{Extração de Informações}

O processo de aquisição de informações do perfil inicia-se a partir do login do usuário no Facebook. Através de sua API (Application Programming Interface), o Facebook disponibiliza informações referentes às conexões de cada usuário, informações públicas do seu perfil, interesses, preferências e ainda grupos dos quais ele participa. As características retiradas como dados de entrada para o sistema de recomendação foram 
V Congresso Brasileiro de Informática na Educação (CBIE 2016)

Anais do XXVII Simpósio Brasileiro de Informática na Educação (SBIE 2016)

selecionadas após a realização de um levantamento de quais elementos poderiam ser relevantes para a criação do modelo do grupo. São elas: nome, descrição, postagens e horário de maior incidência de publicações (características do grupo); e nome, idade, histórico educacional, tipo de mídia mais compartilhada, gênero, idiomas de domínio, interesses, dia e horário de maior acesso, dispositivo usado e as conexões de um usuário com outros usuários (características dos membros do grupo).

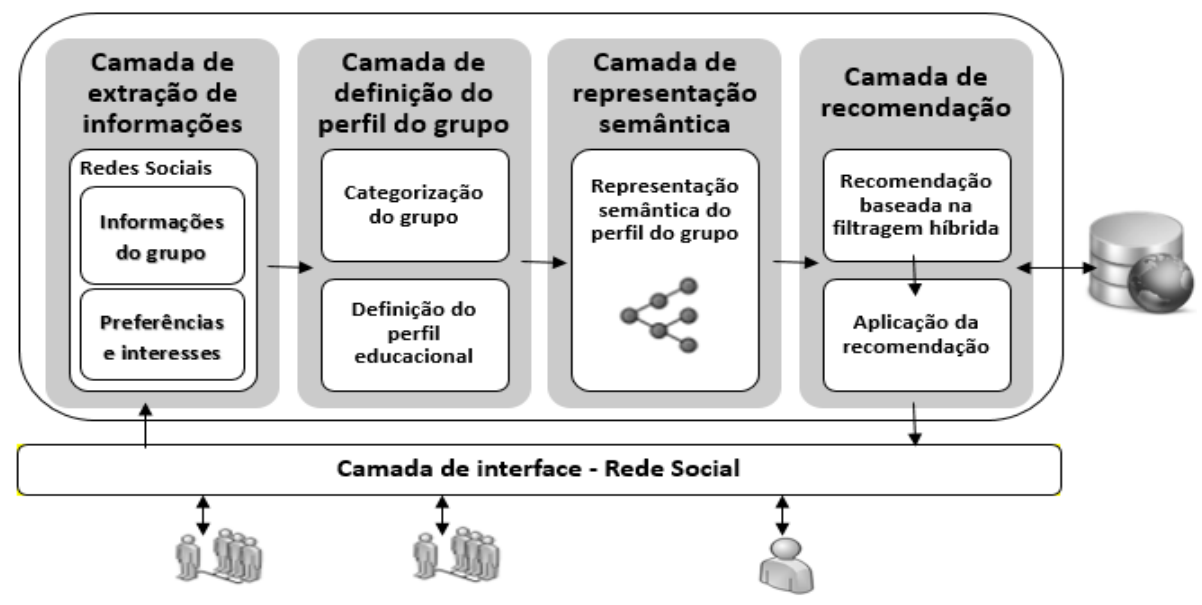

Figura 1. Arquitetura conceitual BROAD-GRS

\subsection{Definição do perfil do grupo}

Depois de extraídas as informações, faz-se necessário filtrá-las, para identificar as de cunho educacional. $O$ Facebook não possui uma categoria explícita com essa informação, como uma alternativa para categorizar o grupo, foi utilizada a AlchemyAPI nas postagens dos usuários. Essa ferramenta oferece um conjunto de funcionalidades sobre o texto fornecido, como extração de tags, detecção de idioma, extração de entidades, categorização de texto, extração de conceitos relacionados, extração de palavras-chave, entre outros. A maioria destas funcionalidades usa métodos estatísticos de processamento de linguagem natural e algoritmos de aprendizagem de máquina. A escolha da AlchemyAPI foi feita por possuir suporte para diferentes idiomas.

Após a categorização do grupo são considerados o perfil educacional do grupo e as áreas de interesse definidas no perfil educacional dos usuários integrantes (tais como páginas referentes a faculdades, escolas, linhas de pesquisa, entre outras). São extraídas, ainda, as preferências por tipos diferentes de mídias, a partir dos quantitativos dos diferentes tipos de recursos compartilhados pelo usuário no Facebook. A preferência é usada para rankear e priorizar um subconjunto de itens recomendáveis.

A proposta aborda a construção de um modelo de grupo estabelecido, ou seja, um grupo onde seus integrantes escolheram explicitamente ser parte deste grupo, devido a algum interesse em comum. A estratégia leva em consideração a maximização da satisfação geral de todos os membros. Para isso, ele analisa as preferências individuais e gera uma categorização de perfil educacional para o grupo. A estratégia de agregação considera apenas as características observadas na maioria dos seus membros, visto que uma característica observada na minoria dos usuários membros não é representativa ao grupo. As características consideradas para a definição do perfil do grupo são: Idiomas falados, preferências por mídias, nível de escolaridade, idade e dispositivo. 
V Congresso Brasileiro de Informática na Educação (CBIE 2016)

Anais do XXVII Simpósio Brasileiro de Informática na Educação (SBIE 2016)

\subsection{Representação Semântica}

A representação semântica do perfil do grupo é feita através de uma ontologia que envolve classes referentes a tópicos de interesse do grupo, instituições onde a pessoa estudou ou trabalhou, grau de formação educacional e preferências por mídias. Esta ontologia representa as informações relacionadas nas redes sociais, fazendo um merge de group com a Sioc (Breslin et al., 2006) e de user com a Foaf (Brickley e Miller, 2015). A Sioc (Semantically-Interlinked Online Communities) fornece os principais conceitos e propriedades necessárias para descrever informações de comunidades online (por exemplo, fóruns, wikis, blogs, etc). Já a Foaf (Friend of a Friend) é uma ontologia que descreve as pessoas, suas ligações e as coisas que podem criar e fazer.

\subsection{Recomendação}

O módulo de recomendação é responsável por realizar buscas em fontes diferentes (no contexto deste trabalho, realiza busca em repositórios de objetos de aprendizagem, conjuntos de dados ligados e repositórios de vídeos), estabelecendo a relação entre o perfil do grupo e os recursos educacionais armazenados nessas fontes. Nesse módulo é definida também a prioridade de recomendação a partir da aderência entre os recursos educacionais e o perfil educacional estabelecido para o grupo.

A recomendação dos recursos educacionais é feita a partir da relação estabelecida entre o perfil do grupo e os metadados do recurso educacional. $O$ uso de dados ligados na recomendação aumenta a possibilidade de encontrar conteúdos educacionais em diferentes fontes que estão sendo constantemente atualizadas, evitando o problema de escassez de novas recomendações (Pereira et al., 2015b). Dentre as iniciativas de disponibilização de conteúdo através de Dados Ligados, são utilizadas, neste trabalho, a DBpedia e a Open University. A recomendação em vídeos se dá através do YouTube. A Proposta utiliza este recurso realizando uma busca, onde são considerados os interesses e o idioma falado pelo usuário. A busca é feita no conjunto de todos os vídeos disponíveis pelo Youtube. Vale ressaltar ainda que a Proposta também pode fazer pesquisas específicas em um determinado Canal, aumentando assim a qualidade e precisão das recomendações educacionais.

\section{Desenvolvimento do Protótipo}

A solução está baseada na linguagem de programação e plataforma computacional Java, desenvolvida na plataforma NetBeans IDE 8.0.2., um ambiente de desenvolvimento integrado gratuito e de código aberto para desenvolvedores de software. Para acessar as informações dos usuários e dos grupos no Facebook foi usada a API fornecida pelo Facebook para desenvolvedores de aplicativos, chamada GraphAPI. Quando alguém se conecta ao Facebook usando um aplicativo, o aplicativo será capaz de obter um token de acesso temporário, permitindo o acesso seguro às APIs do Facebook.

Foi utilizado a AlchemyAPI nas postagens dos usuários como uma alternativa para categorizar o grupo, já que não é possível extrair esta informação através da GraphAPI. É feita a filtragem do texto descritivo das postagens dos usuários no grupo, e, então, esse texto é submetido aos métodos da AlchemyAPI para a detecção do idioma do texto, extração de entidades, conceitos e palavras-chave. Os conceitos e as palavraschave extraídas são retornados juntamente com um grau de relevância. Novamente, 
V Congresso Brasileiro de Informática na Educação (CBIE 2016)

Anais do XXVII Simpósio Brasileiro de Informática na Educação (SBIE 2016)

levando em consideração a maximização da satisfação geral de todos os membros do grupo, foi definido que, para este trabalho, seriam considerados apenas os termos que possuem relevância maior que $50 \%$.

Para trabalhar com as ontologias, criando-as, realizando consultas e manipulando-as foram usados o Apache Jena, o banco de dados RDF Sesame, e a linguagem SPARQL para consultas. Para o armazenamento dos dados de perfil do grupo e de seus usuários membros é utilizado um banco de dados MySQL.

Na recomendação da abordagem baseada em repositório, a Proposta estabelece uma relação entre as características do perfil do grupo e os metadados dos recursos educacionais, através de uma busca por índice usando o Lucene. O Lucene é uma biblioteca Open Source para indexação e consulta de textos, onde os dados originais são indexados gerando uma estrutura de dados relacionados para pesquisa baseada em palavras-chave. Com isso, é feita a determinação da aderência do recurso educacional ao perfil do grupo. Após a realização do cálculo da aderência dos recursos educacionais, e, levando em consideração o índice de similaridade resultante do Lucene, é aplicado o grau de relevância do documento como critério de priorização.

Já na abordagem baseada em Dados Ligados, após ser determinado o tema para qual a recomendação será feita, a extração de recomendações educacionais é realizada buscando por páginas relacionadas ao tema de interesse na DBpedia e na Open University.

A recomendação baseada em vídeos é feita através da API do canal de vídeos Youtube. Esta abordagem é feita indicando quantos vídeos devem ser retornados, e, utilizando apenas as palavras-chave. A própria API do Youtube determina a relevância dos vídeos de acordo com as palavras-chave, ou seja, o vídeo mais relevante de acordo com a API será o primeiro a ser recomendado.

\section{Avaliação do BROAD-GRS}

Estudo de caso é um método empírico que busca investigar fenômenos contemporâneos em seus contextos (Wohlin et al., 2012). A avaliação foi feita através de um estudo de caso que analisou dois grupos de usuários distintos no Facebook. O estudo de caso conduzido tem caráter descritivo e exploratório. A pesquisa descritiva foi empregada na observação dos seguintes fenômenos: (1) Aceitação do usuário em relação às suas características, bem como as características do grupo do qual ele participa, identificadas através de suas redes sociais, no caso, do Facebook. (2) Aceitação dos usuários em relação às recomendações educacionais feitas ao grupo, levando em consideração as características individuais e coletivas do grupo em questão. (3) O repositório de recursos educacionais gerou recomendações satisfatórias aos usuários membros do grupo. (4) Os recursos extraídos de conjuntos de dados ligados geraram recomendações satisfatórias aos usuários membros do grupo. (5) Os vídeos extraídos do Youtube geraram recomendações satisfatórias aos usuários membros do grupo.

Já a pesquisa exploratória foi aplicada com o objetivo de gerar melhorias para o processo de recomendação, na observação do usuário em relação a: (1) Como o usuário analisa o uso de redes sociais para a recomendação de recursos educacionais. (2) Qual a opinião do usuário em relação à Proposta de recomendação e suas funcionalidades. 
Para realizar o estudo de caso foram criados grupos no Facebook, compostos de participantes voluntários. Por questão de espaço relatamos a avaliação de dois subgrupos, denominados Subgrupo 1 e Subgrupo 2. O Subgrupo 1 foi formado por 16 participantes, todos pertencentes a uma escola Técnica de Informática, enquanto o Subgrupo 2, foi formado por 10 participantes de áreas distintas, sendo 6 de Direito, 2 de Engenharia Ambiental e 2 de Ciência da Computação. Um requisito para a seleção dos indivíduos foi possuir uma conta na rede social Facebook. Conforme os usuários iam confirmando a sua participação no Grupo e permitindo que o protótipo tivesse acesso às suas informações pessoais, o perfil educacional do grupo foi sendo definido.

Após finalizar este processo de aceitação e extração de informações implícitas e explícitas, foi definido pelos integrantes um tema para discussão no grupo. $O$ Subgrupo 1 abordou o tema "Wireless Network" enquanto o Subgrupo 2 abordou o tema "Cotas Raciais". Foi adotada a estratégia de recomendar 3 (três) recursos educacionais obtidos através de cada uma das abordagens implementadas para cada grupo de participantes. Não foi possível que o protótipo publicasse os recursos diretamente dentro do Grupo devido às restrições de acesso impostas pela API do Facebook para grupos. No entanto, o protótipo indicou os recursos ao usuário administrador do Grupo, que repassou as informações para os membros de cada subgrupo. A forma que os Dados Ligados são apresentados para os usuários pode ser vista na Figura 2.

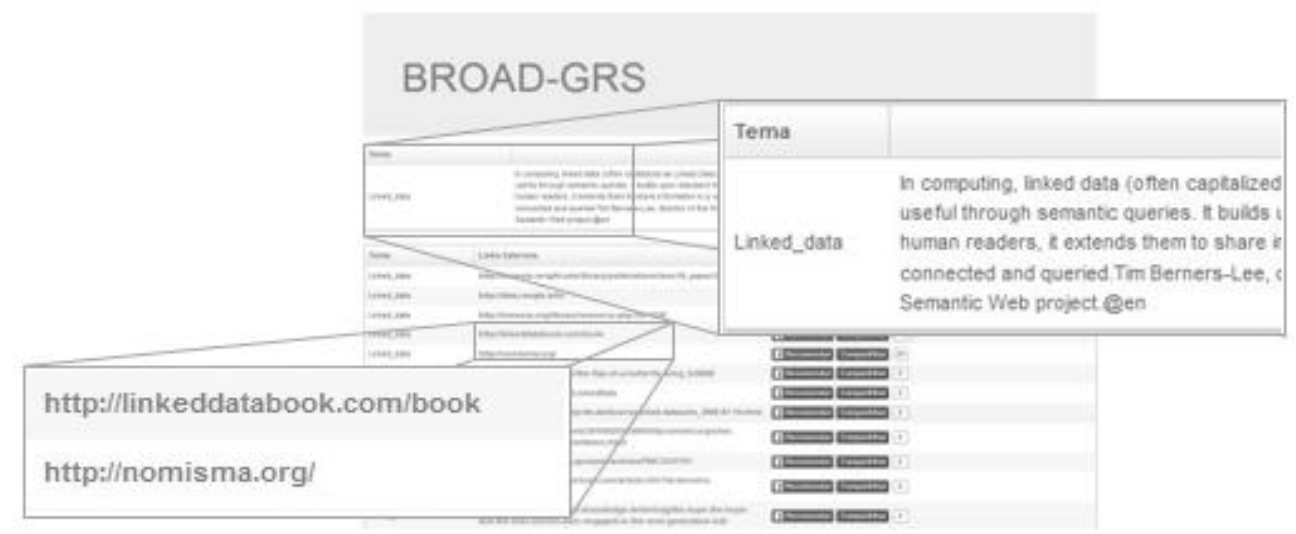

Figura 2. Recomendação com Dados Ligados para o tema "Linked Data"

Os dados utilizados neste estudo de caso foram coletados usando o método direto, através de questionários semiestruturados. Este método é adequado, pois o objetivo é realizar análises quantitativas e qualitativas. Foram usadas perguntas com opções de múltipla escolha para avaliar os dados extraídos do perfil do usuário membro do grupo, do grupo propriamente dito, bem como de seus interesses extraídos, e para os recursos educacionais recomendados no processo. Ainda foram utilizadas perguntas abertas com a finalidade de obter informações não previstas nas perguntas diretas, além de buscar a opinião dos usuários sobre a ferramenta e as suas funcionalidades, e, com isso, obter sugestões de melhoria para o processo de recomendação.

Os participantes do estudo de caso, primeiramente os do Subgrupo 1 e depois os do Subgrupo 2, foram convidados a avaliar os itens extraídos e as recomendações realizadas. O repositório local de recursos educacionais tinha, no momento da avaliação, 74 recursos educacionais relacionados às áreas da Ciência da Computação. A Figura 3 
V Congresso Brasileiro de Informática na Educação (CBIE 2016)

Anais do XXVII Simpósio Brasileiro de Informática na Educação (SBIE 2016)

apresenta o quantitativo de respostas dos usuários a respeito da aceitação dos usuários em relação às recomendações educacionais feitas aos subgrupos, levando em consideração suas características individuais e coletivas do subgrupo em questão.

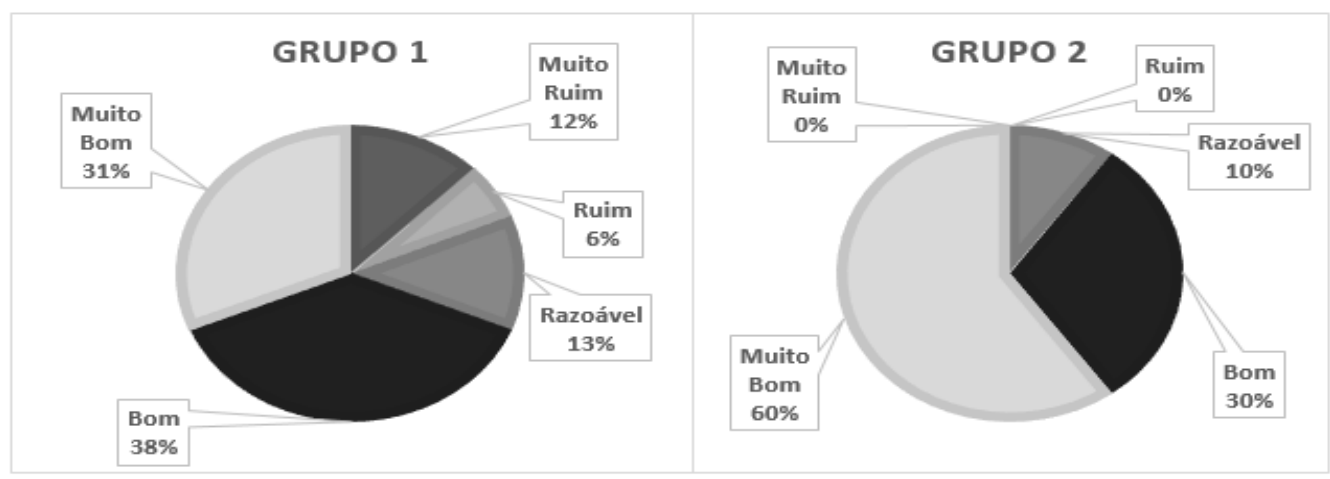

Figura 3. Aceitação dos usuários em relação às recomendações

Os participantes tanto do Subgrupo 1 quanto do Subgrupo 2 avaliaram positivamente o processo de extração de seus dados, com $63 \%$ e $70 \%$, respectivamente, de avaliações "Bom" e "Muito Bom". 69\% dos participantes do Subgrupo 1 também avaliaram positivamente os recursos recomendados ao grupo. Nota-se ainda que o Subgrupo 2 tem uma aceitação alta em termos dos recursos recomendados ao grupo (90\% avaliaram como "Bom" ou "Muito Bom"), mesmo não tendo sido recomendado nenhum arquivo do repositório de objetos de aprendizagem, pois nenhum recurso educacional desta fonte atende a satisfação da maioria dos membros do grupo. A alta aceitação neste caso se deve aos recursos vindos dos dados ligados e dos vídeos.

Análises foram feitas em relação às três fontes de busca dos elementos a serem recomendados. O repositório local da Proposta foi capaz de atender satisfatoriamente ao Subgrupo 1, com 75\% de avaliação positiva (como "Bom" ou "Muito Bom") dos membros. A avaliação positiva também foi observada na recomendação baseada em Dados Ligados, onde o Subgrupo 1 obteve $60 \%$ de satisfação, considerando as avaliações "Bom" e "Muito Bom", enquanto que o Subgrupo 2 apresentou 80\% de avaliações deste tipo. Já nas recomendações em vídeos, os dados são ainda melhores. $\mathrm{O}$ Subgrupo 1 obteve $86 \%$ de satisfação enquanto que o Subgrupo 2 apresentou $90 \%$ de aprovação, considerando as avaliações "Bom" e "Muito Bom".

Os participantes mostraram ainda aprovar o envio de recomendações para as suas redes sociais, conforme pode ser percebido em alguns relatos:

- "Acho um grande mecanismo para ser usado na educação, pois o acesso de crianças e jovens é muito grande. Deveria ser mais explorado, ter incentivo ao público jovem, assim teria bem mais acessos e a educação aconteceria de forma plena, pois passaria a fazer parte da nossa cultura".

- "Acredito ser bastante útil, uma vez que, atualmente, as redes sociais têm grande influência na vida das pessoas. Opiniões têm sido formadas baseadas em conteúdo das redes sociais, dessa forma, o envio de recomendações educacionais vem como um meio de obter informação útil facilmente".

Quanto ao uso da ferramenta, foram coletados depoimentos que experessaram a opinião do participante, inclusive buscando encontrar formas de melhoria e sugestões 
V Congresso Brasileiro de Informática na Educação (CBIE 2016)

Anais do XXVII Simpósio Brasileiro de Informática na Educação (SBIE 2016)

para trabalhos futuros. Esta questão foi de extrema importância, obtendo relatos muito interessantes, como pode ser observado a seguir:

- "Como uma ferramenta de busca demonstrou muito útil, simples de usar, tudo automatizado, mostrando um filtro de seus próprios interesses, e quem não sabe por onde começar uma recomendação, utilizar o perfil como partida é bem interessante".

- "A ideia é muito boa, assim como seu procedimento. Para mim peca um pouco no fator temporal, as páginas relacionadas não tiveram muita relação com a quantidade de acessos/postagens, bem como algumas foram acessadas há muito tempo atrás, não tendo relação com interesses atuais".

\section{Considerações Finais}

A arquitetura da Proposta abordou a definição do perfil educacional de um grupo em redes sociais e a recomendação de recursos educacionais de acordo com o perfil definido para o grupo. O protótipo desenvolvido mostrou que é possível o envio de recomendações educacionais aos usuários membros do grupo, usando esses ambientes para explorar o tempo que os usuários utilizam em redes sociais. O estudo de caso mostrou a viabilidade técnica e a aceitação dos usuários em relação à definição do perfil educacional do grupo do qual os usuários eram membros, e das recomendações feitas.

A abordagem de definição do perfil educacional do grupo na rede social traz avanços na extração de informações geradas dinamicamente dentro do grupo. Além de levar em consideração a preferência dos usuários membros do grupo, permite gerar recomendações de recursos educacionais coerentes com os interesses e as preferências identificadas. A recomendação utilizando as três abordagens (repositório de dados, dados ligados e vídeos) foi considerada satisfatória e complementar. O uso de dados ligados e de um repositório de vídeos no processo de recomendação permitiu ampliar as possibilidades de recomendações oferecidas aos usuários. Além disso, o envio dos recursos educacionais através da recomendação em redes sociais oferece ao usuário a utilização dos recursos de interatividade do ambiente. Ele pode curtir e compartilhar suas experiências sobre um recurso educacional recomendado, aproveitando a facilidade de uso que a rede social oferece em sua utilização e divulgação.

Como trabalhos futuros estamos trabalhando num metamodelo para sistemas de recomendação e projetos de identificação de grupos não formais em redes sociais.

Agradecimentos: à FAPEMIG, CNPq, UFJF e Capes pelo apoio financeiro ao projeto.

\section{Referencias}

Almeida, R. F., Campos, F., Ströele, V., David, M. N. (2015). "Sistemas de Recomendação de Recursos Educacionais para Grupos de Redes Sociais: um Mapeamento Sistemático”. In: XXVI SBIE 2015, Cbie-Laclo 2015, p. 1022-1031.

Breslin, J. G., Decker, S., Harth, A., Bojars, U. (2006). SIOC: an approach to connect web-based communities. International Journal of Web Based Communities - IJWBC v. 2, n. 2, p. 133-142.

Brickley, D., Miller, L. (2015). "FOAF Vocabulary Specification 0.99”. $<$ http://xmlns.com/foaf/spec/\#term_Document $>$, dezembro. 
V Congresso Brasileiro de Informática na Educação (CBIE 2016)

Anais do XXVII Simpósio Brasileiro de Informática na Educação (SBIE 2016)

Carvalho, L., Macedo, H. (2014). "Introdução aos Sistemas de Recomendação para Grupos", In: Revista de Informática Teórica e Aplicada (RITA), Volume 21, Número 1, Páginas: 77-109.

Casagrande, M. F. R., Kozima, G., Willrich, R. (2013). "Técnica de Recomendação Baseada em Metadados para Repositórios Digitais Voltados ao Ensino”. In: II CBIE Congresso Brasileiro de Informática na Educação, p 677-686.

CGI - Comitê Gestor Da Internet No Brasil. (2015). Pesquisa sobre o uso das tecnologias de informação e comunicação nas escolas brasileiras: TIC Educação 2014. 1. ed. São Paulo: p. 1-428.

Junco, R. (2011). The relationship between frequency of Facebook use, participation in Facebook activities, and student engagement. Computers \& Education, 58, p. 162-71.

Park, D. H., Kim, H. K., Choi, I. Y., Kim, J. K. (2012). A literature review and classification of recommender Systems research, Expert Systems with Applications 39, p. 10059-10072.

Pereira, C. K., Campos, F., Ströele, V., David, J. M. N., Braga, R. M. (2014). "Extração de Características de Perfil e de Contexto em Redes Sociais para Recomendação de Recursos Educacionais". In: III CBIE Congresso Brasileiro de Informática na Educação, v. 25, p. 506-515.

Pereira, C. K., Campos, F. C. A., Stroele, V., Braga, R. M., David, J. M. N., Almeida, R. (2015). "Extração de Características de Perfil e de Contexto em Redes Sociais para Recomendação de Recursos Educacionais". Revista Brasileira de Informática na Educação, v. 23, p. 25-39.

Pereira, C. K., Campos, F., Ströele, V., N, J. M., Braga, R. (2015). "Explorando Dados Ligados através de um Sistema de Recomendação Educacional". Anais do XXVI Simpósio Brasileiro de Informática Na Educação (SBIE), p. 1042-1051.

Rezende, P. A. A., Pereira, C. K., Campos, F., David, M. N., Braga, R. (2015). "PERSONNA: proposta de ontologia de contexto e perfil de alunos para recomendação de objetos de aprendizagem". In RBIE Revista Brasileira de Informática na Educação, v.23, n. 01, p.70-84.

Ribeiro, F. A. A., Fonseca, L. C. C., Freitas, M. S. (2013). "Recomendando Objetos de Aprendizagem a partir das hashtags postadas no Moodle". In: II CBIE Congresso Brasileiro de Informática na Educação, p.82-91.

Rodrigues, R. B., Silva, C. M. R, Durão, F. A., Assad, R. E., Garcia, V. C., Meira, S. R. L. (2015). "Um Modelo de Recomendação de Arquivos para Sistemas de Armazenamento em Nuvem". In: XI Simpósio Brasileiro de Sistemas de Informação, p. 111-118.

Wohlin, C., Runeson, P., Höst, M., Ohlsson, M. C., Regnell, B., Wesslén, A. (2012). Experimentation in Software Engineering. Springer Berlin Heidelberg.

Yu, S. J. (2012). The dynamic competitive recommendation algorithm in social network services. Information Sciences 187:1-14. 